\title{
CINÉTICA DE DEGRADAÇÃO DE VITAMINA C EM MANGAS 'PALMER' MINIMAMENTE PROCESSADAS ARMAZENADAS EM DIFERENTES TEMPERATURAS
}

\author{
Kinects of vitamin $\mathrm{C}$ degradation of 'palmer' mangoes (Mangifera indica $\mathbf{L}$.) \\ stored at different temperatures
}

\author{
Juliana Alvarenga Alves ${ }^{1}$, Rita de Cássia Mirela Resende Nassur², Caroline Roberta Freitas Pires², \\ Emanuelle Mara de Alcântara ${ }^{2}$, Juliana Audi Giannoni ${ }^{2}$, Luiz Carlos de Oliveira Lima ${ }^{2}$
}

\begin{abstract}
RESUMO
Conduziu-se este trabalho, com o objetivo de utilizar parâmetros cinéticos para avaliar a degradação de vitamina $\mathrm{C}$ sobre a vida útil de mangas (Mangifera indica L.) minimamente processadas e armazenadas em diferentes temperaturas. Mangas 'Palmer' foram lavadas em água corrente, sanificadas, descascadas, novamente sanificadas e fatiadas manualmente. O produto foi embalado em embalagem de polietileno com tampa e armazenado a $0^{\circ} \mathrm{C}, 6^{\circ} \mathrm{C}$ e $12^{\circ} \mathrm{C}(85-90 \%$ UR). Para o acompanhamento da sua vida útil, a cada 2 dias foram feitas as seguintes análises: valores $\mathrm{L}^{*} \mathrm{a}^{*} \mathrm{e} \mathrm{b}^{*}$, perda de massa, $\mathrm{pH}$, firmeza, sólidos solúveis (SS), acidez titulável (AT) e teor de vitamina $\mathrm{C}$. As mangas minimamente processadas armazenadas à $0^{\circ} \mathrm{C}$ e $6^{\circ} \mathrm{C}$ apresentaram vida útil de 10 dias contra 4 dias das mangas armazenadas à $12^{\circ} \mathrm{C}$. Os dados obtidos por meio de regressão linear com os valores do logaritmo neperiano do teor de ácido ascórbico pelo tempo de armazenagem (dias) mostram que a reação de degradação da vitamina $\mathrm{C}$ se ajusta ao modelo cinético de $1^{\mathrm{a}}$ ordem. O Modelo de Arrhenius foi aplicado às velocidades de reação $(k)$ nas diferentes temperaturas estabelecendo energia de ativação (Ea) de 34,32 $\mathrm{kcal} \mathrm{mol}^{-1}$. A degradação de vitamina $\mathrm{C}$ foi mais lenta $\left(\mathrm{t}_{1 / 2}=63,6\right.$ dias; e $k=0,0109$ dias $\left.^{-1}\right)$ à $0^{\circ} \mathrm{C}$ o que proporcionou maior retenção de seus teores $(89 \%)$ durante 10 dias de armazenamento. As frutas armazenadas à $12^{\circ} \mathrm{C}$ apresentaram maior velocidade de degradação $\left(k=0,1729\right.$ dias $\left.^{-1}\right) \mathrm{e}$, consequentemente, $\mathrm{t}_{1 / 2}$ inferior às demais temperaturas (apenas 4 dias).
\end{abstract}

Termos para indexação: Cinética de degradação da vitamina C, processamento mínimo, Mangifera indica.

\begin{abstract}
This work was used to evaluate the kinetic parameters for degradation of vitamin $\mathrm{C}$ on the shelf-life of minimally processed mangoes (Mangifera indica L.) stored at different temperatures. 'Palmer' Mangos were washed in running water, sanitized, peeled, manually sliced and again sanitized. The product was packaged in polyethylene packaging with lid and stored at $0^{\circ} \mathrm{C}, 6^{\circ} \mathrm{C}$ and $12{ }^{\circ} \mathrm{C}$ (85-90\% RH). To monitor its shelf-life, the following tests were done every two days: values $\mathrm{L}^{*} \mathrm{a}^{*} \mathrm{~b}^{*}$, weight loss, $\mathrm{pH}$, firmness, soluble solids (SS), titratable acidity (TA) and content of vitamin C. The minimally processed mangoes stored at $0^{\circ} \mathrm{C}$ and $6^{\circ} \mathrm{C}$ showed shelf-life of 10 days, whereas the shelf-life of mangoes stored at $12^{\circ} \mathrm{C}$ was 4 days. The data obtained by linear regression with the values of the Neperian logarithm content of ascorbic acid by the time of storage (days) show that the reaction of degradation of vitamin $\mathrm{C}$ fits the kinetic model of $1^{\text {st }}$ order. The Arrhenius model was applied to the speed of reaction $(\mathrm{k})$ at different temperatures by setting the activation energy (Ea) of 34,32 kcal.mol ${ }^{-1}$. The degradation of vitamin $\mathrm{C}$ was slower $\left(\mathrm{t}_{1 / 2}=63.6\right.$ days, and $\mathrm{k}=0.0109$ days ${ }^{-1}$ ) at $0^{\circ} \mathrm{C}$, which provided greater retention of its contents $(89 \%)$ during 10 days of storage. Fruits stored at $12^{\circ} \mathrm{C}$ showed a higher rate of degradation $\left(\mathrm{k}=0.1729\right.$ days $\left.^{-1}\right)$ and, consequently, $\mathrm{t}_{1 / 2}$ less than the other temperatures (only 4 days).
\end{abstract}

Index Terms: kinetics of vitamin C degradation, fresh-cut, Mangifera indica.

(Recebido em 18 de agosto de 2008 e aprovado em 23 de junho de 2009)

\section{INTRODUÇÃO}

A manga (Mangifera indica L.) destaca-se como uma fruta de alto valor comercial em muitas regiões do mundo. Pode ser consumida na forma de sucos, compotas, gelatinas, mas, principalmente, in natura, por apresentar ótima qualidade sensorial e ser rica em vitaminas A e C (Cunha et al., 1994). Mas mangas maduras requerem algumas preparações antes do consumo, como a retirada da casca, a separação da semente e o fatiamento da polpa, o que as tornam bastante adequadas ao processamento mínimo (Rattanapanone \& Watada, 2000).

As perdas pós-colheita de frutas tropicais são um grave problema, em razão da rápida deterioração durante a manipulação, transporte e armazenagem (Yahia, 1998). As transformações que os frutos sofrem, decorrentes do processamento mínimo, pioram o problema, por aumentar a

\footnotetext{
${ }^{1}$ Universidade Federal de Lavras/UFLA - Departamento de Ciência dos Alimentos/DCA - Cx. P. 3037 - $37200-000$ - Lavras, MG juliana_alvarenga@yahoo.com.br

2 Universidade Federal de Lavras/UFLA - Departamento de Ciência dos Alimentos/DCA - Lavras, MG
}

Ciênc. agrotec., Lavras, v. 34, n. 3, p. 714-721, maio/jun., 2010 
atividade metabólica e favorecer a descompartimentalização de enzimas e substratos, causando escurecimento e amolecimento da polpa, deterioração microbiológica e desenvolvimento de sabores e odores indesejados (Ahvenainen, 1996). O processamento resulta em um aumento na respiração e na produção de etileno e, quando associados, aumentam as taxas de outras reações bioquímicas responsáveis por mudanças de cor, odor, textura e qualidade nutricional (Cantwell, 1992). Todas essas mudanças representam um impacto sobre compostos fitoquímicos e sobre as propriedades antioxidantes, esta última atribuída a componentes bioativos tais como vitamina C, E, carotenóides e polifenóis, que são benéficos à saúde e estão presentes no fruto intacto (Robles-Sanchez et al., 2007).

A determinação do conteúdo de ácido ascórbico em vegetais é muito importante, pois além de seu papel fundamental na nutrição humana (Guthrie, 1989), sua degradação pode favorecer o escurecimento não enzimático (Abd Allah et al., 1974), e causar aparecimento de sabor estranho (Bernhardt et al., 1979). Além disso, o ácido ascórbico é um importante indicador, pois sendo a vitamina mais termolábil, sua presença no alimento, indica que, provavelmente, os demais nutrientes também estão sendo preservados (Bender, 1978; Özkan et al., 2004).

Estudos sobre a manutenção da qualidade e o potencial antioxidante em frutas tropicais minimamente processadas são escassos. Os trabalhos publicados, em sua maioria, enfocam o prolongamento da vida-útil. Poucos relataram o efeito do processamento mínimo sobre os componentes bioativos (Robles-Sanchez et al., 2007). É importante considerar que a perda de nutrientes durante a estocagem pode representar fator limitante para o consumo destes produtos (Kabasakalis et al., 2000; Özkan et al., 2004).

Para a previsão da vida útil de alimentos minimamente processados é importante o conhecimento do mecanismo e da cinética das principais reações de deterioração, além da orientação quanto às condições mais adequadas de conservação dos produtos. Assim, neste trabalho, objetivou-se utilizar parâmetros cinéticos para avaliar a degradação de vitamina $\mathrm{C}$ sobre a vida útil de mangas minimamente processadas e armazenadas em diferentes temperaturas.

\section{MATERIAL E MÉTODOS}

As mangas foram adquiridas no mercado local de Lavras, MG, com ausência de injúrias físicas e foram transportadas para o Laboratório de Pós-colheita de Frutas e Hortaliças do Departamento de Ciência dos Alimentos (DCA) da Universidade Federal de Lavras (UFLA), Lavras, MG. As mangas foram lavadas em água corrente com detergente neutro e sanificadas em hipoclorito de sódio $200 \mathrm{mg} \mathrm{L}^{-1}$ por 5 minutos. Em seguida, foram descascadas, manualmente, a polpa separada longitudinalmente do caroço e cortada em pedaços $(30 \mathrm{~mm}$ x $50 \mathrm{~mm}$ ). $\mathrm{O}$ produto minimamente processado foi sanificado em hipoclorito de sódio $50 \mathrm{mg} \mathrm{L}^{-1}$ por 3 minutos. As frutas foram colocadas em peneira plástica para retirada do excesso de líquido por 2 minutos. Em seguida, foram acondicionados (aproximadamente $200 \mathrm{~g}$ ) em embalagens rígidas de polietileno com tampa e armazenadas em câmarafria a $0^{\circ} \mathrm{C} \pm 1 ; 6^{\circ} \mathrm{C} \pm 1$ e $12^{\circ} \mathrm{C} \pm 1(\mathrm{UR} 90 \% \pm 5$ ), por 10 dias e para o acompanhamento da vida-útil das mangas minimamente processadas as seguintes análises foram realizadas a cada 2 dias:

As avaliações de acidez titulável, $\mathrm{pH}$ e sólidos solúveis foram feitas em homogenato filtrado, após trituração das mangas em homogeneizador de tecidos, na proporção 1:5 (manga: água).

Acidez titulável (\% ácido málico) - realizada por titulação com solução de $\mathrm{NaOH} 0,01 \mathrm{~N}$, tendo como indicador fenolftaleína (Instituto Adolfo Lutz, 1985);

pH - utilizou-se pHmetro TECNAL (Tec 3MP), segundo a Association of Official Analytical Chemists-AOAC (2005)

Sólidos solúveis ( ${ }^{\circ}$ Brix) - usou-se refratômetro digital ATAGO PR-100 (AOAC, 2005);

Firmeza (N) - foi realizada no centro de cinco partes das mangas de cada repetição, com auxílio do Texturômetro Stable Micro System modelo TAXT2i, utilizando a sonda tipo agulha $\mathrm{P} / 2 \mathrm{~N}$ ( $2 \mathrm{~mm}$ de diâmetro), que mediu a força de penetração desta nas partes, numa velocidade de $10 \mathrm{~mm} / \mathrm{s}$ e numa distância de penetração de $3 \mathrm{~mm}$.

Valores $\mathbf{L}^{*} \mathbf{a}^{*} \mathbf{e} \mathbf{b}^{*}$ - as leituras foram feitas no centro de cinco partes de cada pedaço de manga minimamente processada, usando-se colorímetro marca Minolta, modelo CR 400, com a determinação no sistema CIE L*a*b* e com iluminante $\mathrm{D}_{65}$.

Perda de massa (\%) - calculada pela diferença entre a massa inicial dos produtos minimamente processados dentro das embalagens e a obtida em cada intervalo de armazenamento, utilizando balança semianalítica Mettler modelo PC2000.

Vitamina $\mathrm{C}$ total (mg de ácido ascórbico. $100 \mathrm{~g}^{-1}$ de polpa) O teor de ácido ascórbico (após a oxidação a ácido dehidroascórbico) foi determinado pelo método 
colorimétrico, utilizando-se 2,4-dinitrofenilhidrazina, segundo Strohecker \& Henning (1967).

$\mathrm{O}$ experimento foi conduzido em delineamento inteiramente casualizado (DIC), em que os tratamentos foram constituídos por três temperaturas $\left(0,6\right.$ e $\left.12^{\circ} \mathrm{C}\right)$ seis tempos de armazenamento (0, 2, 4, 6, 8 e 10 dias), com 3 repetições. A parcela experimental foi constituída por uma embalagem contendo cerca de $200 \mathrm{~g}$ do produto minimamente processado. As análises estatísticas foram feitas utilizando-se o software Sisvar (Ferreira, 2000).

Parâmetros cinéticos - Os dados obtidos da concentração de vitamina $\mathrm{C}$ para cada temperatura foram analisados quanto à ordem de reação e calculadas as respectivas constantes de reação $(\mathrm{k})$, energia de ativação (Ea) e o tempo de meia vida $\left(\mathrm{t}_{1 / 2}=\ln 2 / \mathrm{k}\right)$ conforme Labuza (1982).

Para a determinação da ordem de reação e sua constante de velocidade, foram plotados os valores de teor de vitamina $\mathrm{C}$ (mg ácido ascórbico. $100 \mathrm{~g}^{-1}$ polpa) versus tempo de armazenamento para cada temperatura, e realizada a regressão linear desses valores. A inclinação obtida na regressão linear, de cada temperatura, corresponde aos valores de $k$ (constante de velocidade da reação).

Para a dependência da velocidade da reação com a temperatura, foi utilizado o Modelo de Arrhenius. A inclinação da reta de $\ln (k)$ versus $1 / \mathrm{T}\left(\mathrm{K}^{-1}\right)$ é conhecida como $\mathrm{Ea} / \mathrm{R}$. Sendo $\mathrm{R}=1,987 \mathrm{cal} / \mathrm{gmol} \mathrm{K}$, determinou-se a energia de ativação (Ea). Por meio da equação $(\ln k / 2)$ calculou-se o tempo de meia vida, $\mathrm{t}_{1 / 2}$ da reação de degradação da vitamina C. O tempo de meia vida se refere ao tempo necessário para que a concentração de vitamina $\mathrm{C}$ se reduza à metade do valor inicial.

\section{RESULTADOS E DISCUSSÃO}

As mangas minimamente processadas armazenadas à $0^{\circ} \mathrm{C}$ e $6^{\circ} \mathrm{C}$ apresentaram-se próprias para o consumo durante 10 dias de armazenamento, contra 4 dias das mangas armazenadas à $12^{\circ} \mathrm{C}$.

As variáveis valor $\mathrm{a}^{*} \mathrm{e} \mathrm{pH}$ não foram afetadas estatisticamente nem pelo tempo de armazenamento, nem pela temperatura, nem tampouco pela interação desses dois fatores em mangas minimamente processadas. $\mathrm{O}$ valor $\mathrm{a}^{*}$ das mangas manteve-se em média $-2,53$ e o valor médio do $\mathrm{pH}$ em mangas armazenadas em diferentes temperaturas foi 4,0 .

Houve influência do tempo de armazenamento sobre as variáveis perda de massa, firmeza, acidez titulável, valor $L^{*}$ e valor $b^{*}$. Não se observou influência da temperatura, nem da interação entre a temperatura e o tempo de armazenamento sobre essas variáveis.
As porcentagens de perda de massa das mangas minimamente processadas aumentaram gradualmente ao longo do armazenamento, não ultrapassando $0,50 \%$ (Figura 1A). Melo \& Vilas Boas (2007), estudando a redução do amaciamento de banana 'maçã' minimamente processada pelo uso de tratamentos químicos, também observaram uma perda de massa mínima, que atingiu um máximo de 0,45\%. O mesmo foi observado por Alves et al. (2009) em um 'mix' de cenoura, abóbora, mandioquinha salsa e chuchu, minimamente processados, em que a perda de massa apresentou um pequeno incremento durante o armazenamento. Esta baixa perda de massa é considerada aceitável, do ponto de vista prático. Segundo Chitarra \& Chitarra (2005), frutas e hortaliças, mesmo quando mantidas em condições ideais, podem apresentar perda de massa durante o armazenamento, em razão do efeito combinado da respiração e da transpiração.

As mangas minimamente processadas apresentaram pequena redução na firmeza de $0,489 \mathrm{~N}$ para $0,268 \mathrm{~N}$, durante 10 dias de armazenamento (Figura 1B). A diminuição da firmeza está relacionada ao processo de amaciamento dos tecidos, decorrente de modificações na estrutura e composição da parede celular pela ação de enzimas. Portanto, a degradação da celulose, das hemiceluloses e das pectinas das paredes celulares é o principal fator responsável pela perda de firmeza dos tecidos vegetais, após o processamento mínimo (Chitarra \& Chitarra, 2005).

Normalmente, os ácidos orgânicos tendem a diminuir no decorrer do armazenamento, à medida que são utilizados durante a respiração ou convertidos em açúcares, como o que acontece com pequis minimamente processados e armazenados a $6^{\circ} \mathrm{C}$ durante 15 dias. Contudo, podem aumentar, como ocorre em banana e abacaxi, atingindo altos níveis no estádio pleno de amadurecimento (Damiani et al., 2008) assemelhando-se ao comportamento da acidez dos frutos aqui pesquisados (Figura 1C). A acidez titulável aumentou durante o armazenamento de mangas minimamente processadas, provavelmente em decorrência do baixo metabolismo respiratório que gerou um acúmulo de ácidos nos vacúolos, à medida que os teores de sólidos solúveis foram aumentando. Oliveira et al. (2007), estudando o efeito do corte nas características físico-químicas de melão 'cantaloupe' minimamente processado, também observaram aumento nos valores de acidez titulável no decorrer do armazenamento.

Com relação à coloração da manga minimamente processada observou-se aumento no valor $\mathrm{L}^{*}$ e diminuição do valor $b^{*}$ durante o armazenamento. $\mathrm{O}$ valor $\mathrm{L}^{*}$ 

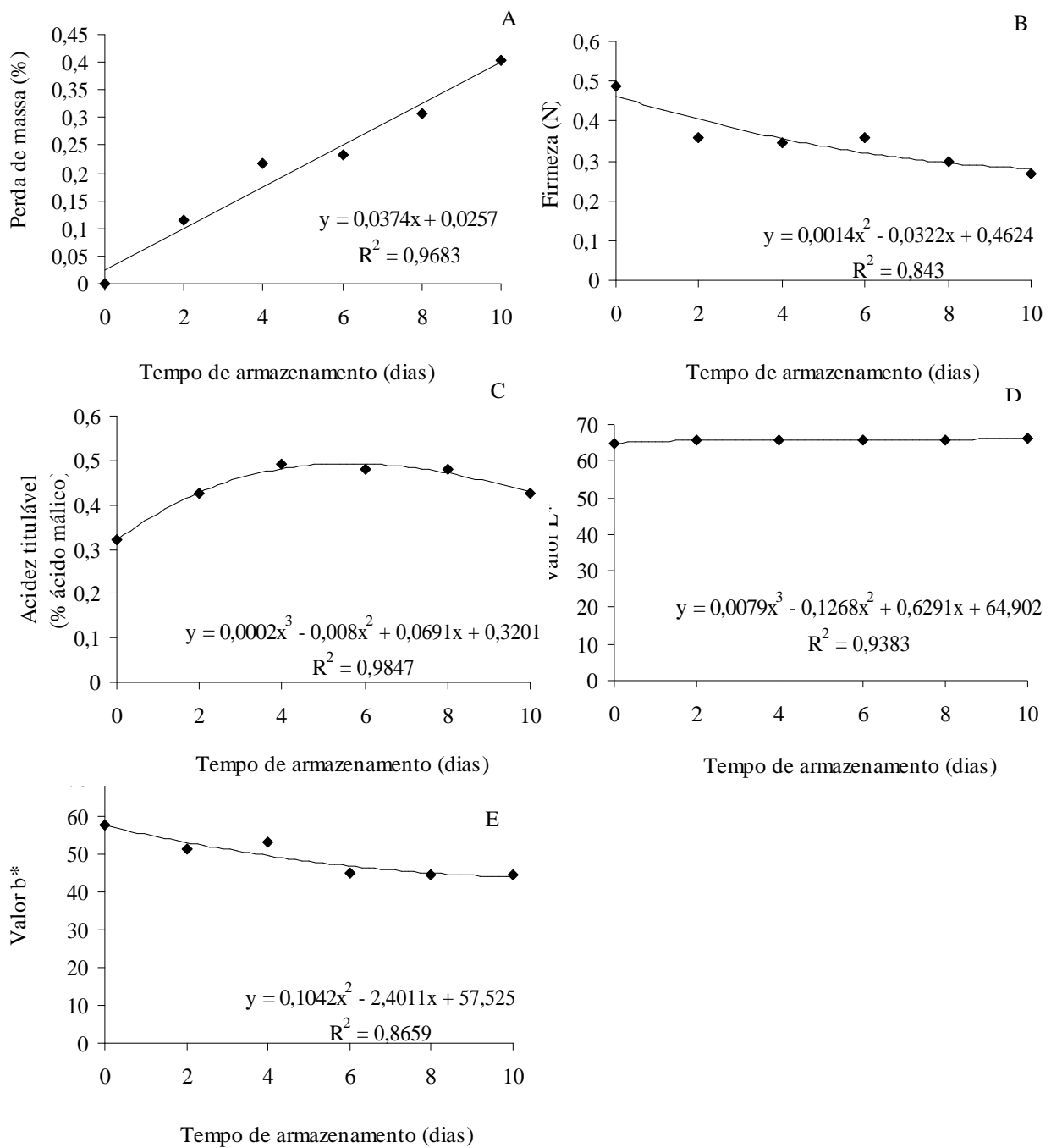

Figura 1 - Equação de regressão e coeficiente de determinação da perda de massa (A), firmeza (B); acidez titulável (C); valor $\mathrm{L}^{*}(\mathrm{D})$ e valor $\mathrm{b}^{*}(\mathrm{E})$ em mangas 'Palmer' minimamente processadas armazenadas em três temperaturas: $0^{\circ} \mathrm{C}, 6^{\circ} \mathrm{C}$ e $12^{\circ} \mathrm{C}$ por 10 dias.

apresentou um ligeiro incremento, ao longo do armazenamento, de 64,95 para 66,47, indicando aumento de claridade, ou seja, esbranquiçamento da manga minimamente processada (Figura 1D). Isso acontece em decorrência da deposição de lignina na superfície da área cortada, que está relacionada ao processo de cicatrização do ferimento (Bolin \& Huxsoll, 1991). A redução do valor b* (Figura 1E) indica que houve uma diminuição da coloração amarela da polpa, decorrente dos cortes sofridos, podendo ocorrer oxidação enzimática, por meio da interação entre substratos e enzimas e, também, por degradação de vitaminas, principalmente os carotenóides que conferem pigmentação que variam do amarelo ao vermelho, sobretudo o beta-caroteno que está relacionado com a pigmentação amarela.

A variável sólidos solúveis (Figura 2) foi influenciada significativamente pela interação entre os fatores temperatura e tempo de armazenamento. No dia do processamento, o teor de SS nos frutos foi de $9,66^{\circ}$ Brix, enquanto que no último dia de armazenamento o teor máximo foi de $13,66,14,66$ e $15,66{ }^{\circ}$ Brix para as temperaturas de $0^{\circ} \mathrm{C}, 6^{\circ} \mathrm{C}$ e $12^{\circ} \mathrm{C}$, respectivamente. De acordo com Vilas-Boas \& Lima (1999), o acúmulo de açúcares, durante a vida útil dos vegetais, pode ocorrer 
em decorrência da conversão do amido em açúcares. O aumento no teor de SS pode vir também da síntese de compostos secundários como fenólicos simples, em resposta às etapas do processamento mínimo (Chitarra \& Chitarra, 2005) e também pelo acúmulo de ácidos orgânicos.

Observou-se efeito significativo da interação dos fatores tempo de armazenamento e temperatura sobre o teor de vitamina $\mathrm{C}$ das mangas minimamente processadas, que apresentaram redução ao longo do armazenamento (Figura 3). Segundo Chitarra \& Chitarra (2005), o teor de vitamina $\mathrm{C}$ tende a diminuir com o armazenamento, pois os danos mecânicos causados pelo processamento mínimo nos tecidos promovem a desorganização celular ocasionando a oxidação do ácido ascórbico, em razão da atuação direta da enzima ácido ascórbico oxidase (ascorbinase), ou pela ação de enzimas oxidantes como a peroxidase.

Houve diferença significativa no teor de vitamina $\mathrm{C}$ entre as mangas armazenadas à $12^{\circ} \mathrm{C}$ daquelas armazenadas a $0^{\circ} \mathrm{C}$ e $6^{\circ} \mathrm{C}$ no quarto dia de armazenamento. Constatou-se redução de $10,5 \%$ e $27 \%$ no teor de ácido ascórbico entre os dias 0 e 10 na temperatura de $0^{\circ} \mathrm{C}$ e $6^{\circ} \mathrm{C}$, respectivamente. $\mathrm{Na}$ temperatura de $12^{\circ} \mathrm{C}$ a redução no teor de ácido ascórbico foi de 49,9\% no quarto dia de armazenamento, quando essas frutas apresentaram perda de firmeza tornando-se impróprias para o consumo.

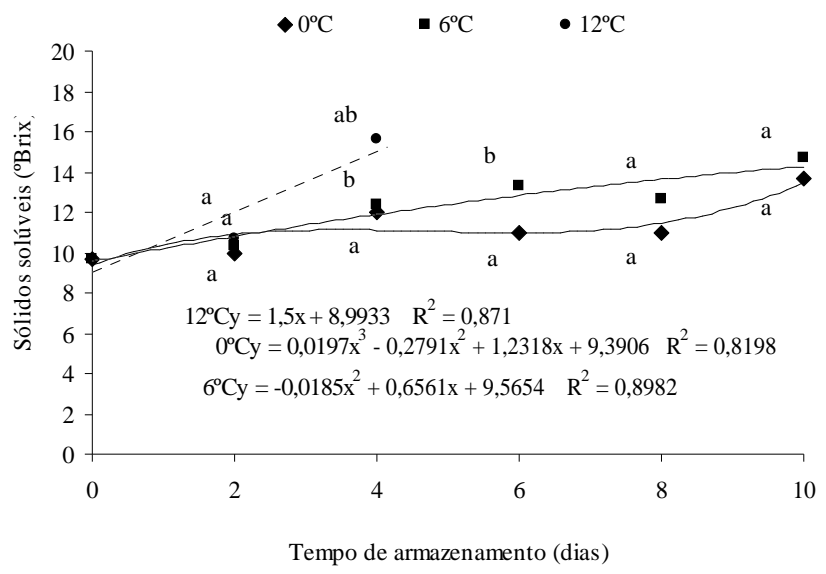

Figura 2 - Equação de regressão e coeficiente determinação de sólidos solúveis em mangas 'Palmer' minimamente processadas armazenadas à $0^{\circ} \mathrm{C}, 6^{\circ} \mathrm{C}$ e $12^{\circ} \mathrm{C}$ por 10 dias. Médias seguidas da mesma letra, dentro de cada tempo, não diferem estatisticamente entre si, pelo Teste Tukey, a $5 \%$.

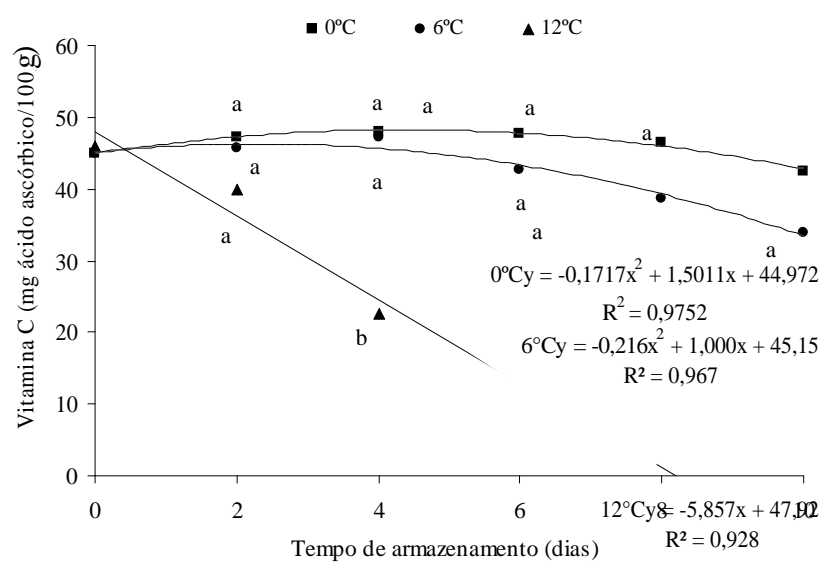

Figura 3 - Equação de regressão e coeficiente de determinação de vitamina $\mathrm{C}$ em mangas 'Palmer' minimamente processadas armazenadas em três temperaturas: $0^{\circ} \mathrm{C}, 6^{\circ} \mathrm{C}$ e $12^{\circ} \mathrm{C}$ por 10 dias. Médias seguidas da mesma letra, dentro de cada tempo, não diferem estatisticamente entre si, pelo Teste Tukey, a $5 \%$.

Ciênc. agrotec., Lavras, v. 34, n. 3, p. 714-721, maio/jun., 2010 


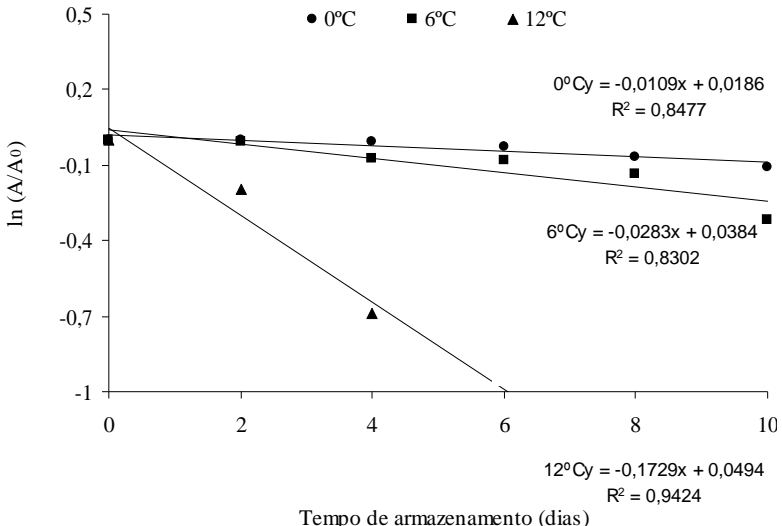

Tempo de armazenamento (dias)

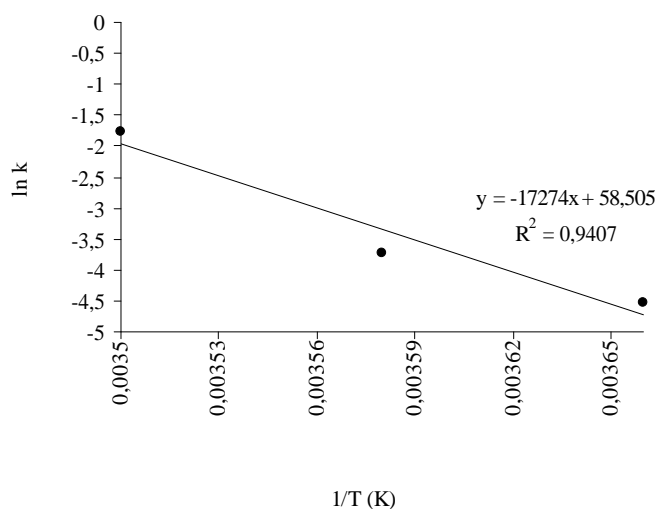

$1 / \mathrm{T}(\mathrm{K})$

Figura 4 - Degradação de vitamina $\mathrm{C}$ em função do tempo sob diferentes temperaturas (A) e Gráfico de Arrhenius para o teor de vitamina $\mathrm{C}(\mathrm{B})$.

Estudos em manga fresca minimamente processada (Rattanapanone et al., 2001; Martinez-Ferrer et al., 2002; Plotter et al., 2004; Gil et al., 2006) demonstraram que o armazenamento a frio $\left(0-5^{\circ} \mathrm{C}\right)$ na atmosfera controlada e/ou modificada, tratados com estabilizadores retentores de cor e firmeza, pode prolongar a vida útil de até 10 dias sem aparente alteração da sua capacidade comercial, mas com perdas de $5 \%$ em vitamina $\mathrm{C}$.

Os dados obtidos por meio de regressão linear com os valores do logaritmo neperiano do teor de ácido ascórbico pelo tempo de armazenamento (dias) mostram que a reação de degradação de vitamina $\mathrm{C}$ se ajusta ao modelo cinético de $1^{\mathrm{a}}$ ordem (Figura 4A). Segundo Atkins \& Paula (2004), em uma reação de primeira ordem, a concentração do reagente diminui exponencialmente com o tempo, numa velocidade determinada pela constante $k$.

Esse comportamento tem sido reportado como característico de vitamina $\mathrm{C}$, que em geral apresenta cinética de degradação de primeira ordem (Villota \& Hawkes, 1992). Nos alimentos, a maioria das reações que representam importantes perdas de qualidade é caracterizada por modelos de ordem zero (qualidade de alimentos congelados, escurecimento não enzimático) ou modelos de primeira ordem (perda de vitaminas, crescimento ou morte de microrganismos, oxidação da cor, perda de textura em alimentos termicamente processados) (Taoukis et al., 1997).

Os parâmetros cinéticos para a degradação de vitamina $\mathrm{C}$ em mangas minimamente processadas encontram-se na Tabela 1. O Modelo de Arrhenius (Figura 4B) foi aplicado às constantes de velocidades da reação
( $k$ ) nas diferentes temperaturas estabelecendo uma energia de ativação (Ea) de 34,32 kcal.mol ${ }^{-1}$. A degradação de vitamina $\mathrm{C}$ foi mais lenta $\left(k=0,0109 \operatorname{dias}^{-1}\right)$ à $0^{\circ} \mathrm{C}$ o que proporcionou maior retenção de seu teor $(89 \%)$ durante 10 dias de armazenamento. As frutas armazenadas à $12^{\circ} \mathrm{C}$ apresentaram maior constante de velocidade de degradação $\left(k=0,1729 \operatorname{dias}^{-1}\right)$.

Os valores de tempo de meia-vida, $\mathrm{t}_{1 / 2}$, confirmam que o teor de vitamina $\mathrm{C}$ das mangas minimamente processadas decresce quando estas são armazenadas em temperaturas mais altas (Tabela 1). Considerando que o tempo de meia-vida é intervalo de tempo necessário para a concentração inicial de um reagente cair à metade do seu valor inicial (Atkins \& Paula, 2004), as frutas armazenadas à $12^{\circ} \mathrm{C}$ requereram 4 dias para alcançar esta condição, enquanto que as frutas armazenadas à $6^{\circ} \mathrm{C} \mathrm{e}$ $0^{\circ} \mathrm{C}$ requereram aproximadamente 29 e 64 dias, respectivamente, ou seja, as condições de processamento e a alta temperatura de armazenamento aceleraram a reação de degradação do ácido ascórbico. Segundo Bobbio \& Bobbio (2003), sua estabilidade aumenta com o abaixamento da temperatura.

Tabela 1 - Parâmetros cinéticos da degradação de vitamina $\mathrm{C}$ em manga minimamente processada armazenada em diferentes temperaturas: $0^{\circ} \mathrm{C}, 6^{\circ} \mathrm{C}$ e $12^{\circ} \mathrm{C}$, por 10 dias.

\begin{tabular}{cccc}
\hline & \multicolumn{3}{c}{ Temperatura } \\
\cline { 2 - 4 } Parâmetros cinéticos & $0^{\circ} \mathrm{C}$ & $6^{\circ} \mathrm{C}$ & $12^{\circ} \mathrm{C}$ \\
\hline Ordem de reação & $1^{\mathrm{a}}$ & $1^{\mathrm{a}}$ & $1^{\mathrm{a}}$ \\
$k\left(\right.$ dias $\left.^{-1}\right)$ & 0,0109 & 0,0238 & 0,1729 \\
$\mathrm{~T}_{1 / 2}($ dias $)$ & 63,57 & 29,12 & 4,01 \\
$\mathrm{Ea}\left(\mathrm{kcal} \mathrm{mol}^{-1}\right)$ & & 34,32 & \\
\hline
\end{tabular}




\section{CONCLUSÕES}

Durante o período em que as mangas mantiveramse próprias para o consumo, a perda de ácido ascórbico foi inferior a 50\% do valor inicial, o que demonstra que o teor de vitamina $\mathrm{C}$ é um bom parâmetro para acompanhar a vida útil de mangas minimamente processadas.

\section{AGRADECIMENTOS}

Agradecimentos à FAPEMIG (Fundação de Amparo à Pesquisa do Estado de Minas Gerais) pelas bolsas de estudos.

\section{REFERÊNCIAS BIBLIOGRÁFICAS}

ABD ALLAH, M.A.; ZAKI, M.S.A. Preservation of mango juice by freezing and canning. Lie Narung, v.18, p.207-16, 1974.

AHVENAINEN, R. New approaches in improving the shelf life of minimally processed fruit and vegetables. Trends in Food Science Technology, Cambridge, v.7, p.179-187, 1996.

ALVES, J. A.; VILAS BOAS, E. V. de B.; SOUZA, E. C. de; VILAS BOAS, B. M.; PICCOLI, R. H. Vida útil de produto minimamente processado composto por abóbora, cenoura, chuchu e mandioquinha-salsa. Ciência e Agrotecnologia, Lavras, v.34, n.1, p. 182-189, jan./fev., 2010.

\section{ASSOCIATION OF OFFICIAL ANALYTICAL} CHEMISTS. Official methods of analysis of analysis of association of official analytical chemists. 18.ed. Maryland, 2005.

ATKINS, P.; PAULA, J. de. Atkins: fisico-quimica. 7.ed. Rio de Janeiro: LTC, 2004. 2v.

BENDER, A.E. Food processing and nutrition. London: Academic, 1978. 243p.

BERNHARDT, L.W. et al. Mudanças que ocorrem durante o armazenamento de frutas e hortaliças congeladas. Boletim do Instituto de Tecnologia de Alimentos, Campinas, v.16, p.9-34, 1979.

BOBBIO, F.O.; BOBBIO, P.A. Introdução à química de alimentos. 3.ed. São Paulo: Varela, 2003. 238p.

BOLIN, H.R.; HUXSOLL, C.C. Effect of preparation and storage parameters on quality retention of salad-lettuce. Journal of Food Science, Chicago, v.56, p.67, 1991.
CANTWELL, F.F.M. Postharvest handling systems: minimally processed fruits and vegetables. In: KADER, A.A. (Ed.). Postharvest technology of horticultural crops. 2.ed. Davis: Academy, 1992. p.277-281.

CHITARRA, M.I.F.; CHITARRA, A.B. Pós-colheita de frutas e hortaliças: fisiologia e manuseio. 2.ed. rev. e ampl. Lavras: UFLA, 2005. 785p.

CUNHA, G.A.P. da; SAMPAIO, J.M.M.; NASCIMENTO, A.S.; SANTOS FILHO, H.P.; MEDINA, V.M.Manga para exportação: aspectos técnicos da produção. Brasília: EmbrapaSPI, 1994. 35p. (Série Publicações Técnicas FRUPEX, 8).

DAMIANI, C.; VILAS-BOAS, E.V.B.; PINTO, D.M.; RODRIGUES, L.J. Influência de diferentes temperaturas na manutenção da qualidade de pequi minimamente processado. Ciência e Agrotecnologia, Lavras, v.32, n.1, p.203-212, jan./fev. 2008.

FERREIRA, D.F. Análises estatísticas por meio do SISVAR para windows versão 4.0. In: REUNIÃO ANUAL DA REGIÃO BRASILEIRA DA SOCIEDADE

INTERNACIONAL DE BIOMETRIA, 45., 2000, São Carlos. Programa e Resumos... São Carlos: UFSCar, 2000. p.235.

GIL, M.I.; AGUAYO, E.; KADER, A.A. Quality changes and nutrient retention in fresh-cut versus whole fruits during storage. Journal of Agricultural Food

Chemistry, Easton, v.54, p.4284-4296, 2006.

GUTHRIE, H.A. Introductory nutrition. 7.ed. Saint Louis: Mosby, 1989. 394p.

INSTITUTO ADOLFO LUTZ. Normas analíticas, métodos químicos e físicos para análise de alimentos. 3.ed. São Paulo, 1985. v.1, 533p.

KABASAKALIS, V.; SIOPIDOU, D.; MOSHATOU, E. Ascorbic acid content of commercial fruit juices and its rate of loss upon storage. Food Chemistry, Chicago, v.70, n.3, p.325-328, Aug. 2000.

LABUZA, T.P. Shelf-life dating of foods. Westport: Food \& Nutrition, 1982.

MARTÍNEZ-FERRER, M.; HARPER, C.; PÉREZ-MUÑOZ, F.; CHAPARRO, M. Modified atmosphere packaging of minimally processed mango and pineapple fruits. Journal of Food Science, Chicago, v.67, p.3365-3371, 2002. 
MELO, A. A. M.; VILAS BOAS, E. V. de B. Redução do amaciamento de banana 'maçã' minimamente processada pelo uso de tratamento químicos. Ciência e

Agrotecnologia, Lavras, v.31, n. 3, p.821-828, maio/jun., 2007.

OLIVEIRA, A. C. de; FIGUEIREDO, R. de W.; MAIA, G. A.; ALVES, R. E; FILHO, M. de S. M. de S.; SOUSA, P. H. M. de. Efeito do tipo de corte nas características físico-químicas e microbiológicas de melão 'cantaloupe' (Cucumis melo L. Híbrido hy-Mark) minimamente processado. Ciência e Agrotecnologia, Lavras, v. 31, n. 4, p. 1095-1101, jul./ago., 2007.

ÖZKAN, M.; AYSEGÜL, K.; CEMEROGLU, B. Effects of hydrogen peroxide on the stability of ascorbic acid during storage in various fruit juices. Food Chemistry, Chicago, v.88, n.4, p.591-597, Dec. 2004.

RATTANAPANONE, N.; WATADA, A.E. Respiration rate and respiration quocient of fresh-cut mango (Mangifera indica L.) in low oxygen atmosphere. Acta Horticultureae, Wageningen, n.509, p.471-478, 2000.

RATTANAPANONE, N.; LEE, Y.; WU, T.; WATADA, A.E. Quality and microbial changes of fresh-cut mango cubes held in controlled atmosphere. Hortscience, Alexandria, v.36, p.1091-1109, 2001.
ROBLES-SANCHEZ, M.; GORINSTEIN, S.; MARTINBELLOSO, O. Minimal processing of tropical fruits: antioxidant potential and its impact on human health. Food Chemistry, Chicago, v.32, n.4, p.227-232, 2007.

STROHECKER, R.; HENNING, H.M. Analisis de vitaminas: métodos comprobados. Madrid: Paz Montalvo, 1967. 428p.

TAOUKIS, P.S.; LABUZA, T.P.; SAGUY, I.S. Kinetics of food deterioration and shelf-life prediction. In: VALENTAS, K.J.; ROTSTEIN, E.; SINGH, R.P. The handbook of food engineering practice. Boca Raton: CRC, 1997. p.361-402.

VILAS-BOAS, E.V.B.; LIMA, L.C.O. Armazenamento de abacaxi 'Pérola'. In: SIMPÓSIO LATINO AMERICANO DE CIÊNCIA DOS ALIMENTOS, 3., 1999, Campinas.

Resumos... Campinas: Unicamp, 1999. p.45.

VILLOTA, R.; HAWKES, J.G. Reaction kinetics in food systems. In: HELDMAN, D.R.; LUND, D.B. (Ed.).

Handbook of food engineering. New York: M.Dekker, 1992. p.39-144.

YAHIA, E. Modified and controlled atmospheres for tropical fruits. Horticultural Reviews, New York, v.22, p.123-183, 1998. 